\title{
THE ROLE OF DISAGREEMENT IN SEMANTIC THEORY
}

\author{
Carl Baker (c.baker@abdn.ac.uk) \\ Northern Institute of Philosophy, University of Aberdeen
}

This is a preprint of an article whose final and definitive form will be published in the Australasian Journal of

Philosophy 2014; the Australasian Journal of Philosophy is available online at: http: / /www.tandf.co.uk/journals/.

\begin{abstract}
Arguments from disagreement often take centre stage in debates between competing semantic theories. This paper explores the theoretical basis for arguments from disagreement and, in so doing, proposes methodological principles which allow us to distinguish between legitimate arguments from disagreement and dialectically ineffective arguments from disagreement. In the light of these principles I evaluate Cappelen \& Hawthorne's (2009) argument from disagreement against relativism, and show that it fails to undermine relativism since it is dialectically ineffective. Nevertheless, I argue that an alternative challenge to relativism based on disagreement is available. More generally, I argue that semantic theory is not answerable to data stemming from 'loaded' philosophical principles regarding the nature of disagreement. Rather, semantic theorists will exhaust their dialectical responsibilities regarding disagreement if they can demonstrate consistency with a minimal account of the concept.
\end{abstract}

Arguments from disagreement often play an influential role in debates between semantic theories. In the recent debate over relativism, for instance, arguments from disagreement have been variously taken to provide evidence either for or against different forms of semantic relativity in expressions such as taste predicates and epistemic modals. This paper explores an aspect of these arguments which has not yet received the attention it deserves: namely, the role of philosophical assumptions about the nature of disagreement in arguments from disagreement. I explore what an argument from disagreement may legitimately assume about disagreement's nature whilst remaining dialectically effective in a debate over semantic theory - that is, to establish what should be considered data to be accommodated by a successful semantic theory, and the extent to which substantive philosophical assumptions about disagreement can be considered part of this data. The aim is not only to establish methodological constraints on the use of arguments from disagreement in semantic theory, but also to shed light on how we should theorise about disagreement more generally. ${ }^{1}$

\footnotetext{
${ }^{1}$ I focus solely on the notion of disagreement as a state, rather than as an activity (Cappelen \& Hawthorne (2009: 60-61)). A state of disagreement is most naturally understood as a passive concept, arising out of some conflict between subjects' attitudes, and not necessarily involving any active behavioural features such as the presence of a dispute. In the stative sense, two subjects might disagree (e.g. in virtue of inconsistency between their beliefs) even though neither subject is aware of the existence of the state.
} 
Section one gives an example of how one can effectively appeal to disagreement in the debate over the semantics of taste-predicates - specifically, by outlining an argument from disagreement against contextualism, and noting that this argument crucially depends on a certain principle regarding the nature of disagreement. Section two distinguishes the argument against contextualism from a further (fictional) argument against a different view — an argument which rests on a clearly illicit assumption regarding disagreement. In contrasting the two arguments, and thereby diagnosing why the assumption is illicit, I derive methodological principles for the use of arguments from disagreement in semantic theory. Sections three and four apply these principles to Cappelen \& Hawthorne's argument from disagreement against relativism. I show that their argument suffers from the same dialectical deficiencies as the fictional argument discussed in section two. In defending this position, an argument which anticipates my concerns is considered and rejected. Nevertheless, I argue that - despite the failure of Cappelen \& Hawthorne's argument - an alternative challenge to relativism based on disagreement is available. In concluding, I draw broader morals about the role of philosophical assumptions regarding disagreement in semantic theory and the extent to which these assumptions can be considered part of the data for semantic theorising.

\section{Arguments From Disagreement In the Debate Over Relativism}

Consider the following exchange:

(1) Bob: 'Spinach is tasty.'

Carol: 'Spinach is not tasty.'

Assuming that Bob and Carol are sincere, this appears to be a paradigm case of disagreement about taste. This observation has been taken to cause trouble for certain semantic treatments of predicates like 'tasty' - and contextualist theories often bear the brunt of such objections. ${ }^{2}$ Contextualists hold that sentences like 'Spinach is tasty' are context-sensitive, in that the content they express varies between contexts of use. Simple forms of this view hold that the contextsensitivity in question arises from the varying standards of taste of speakers across contexts. For

\footnotetext{
${ }^{2}$ This argument has also been formulated with respect to other areas of discourse. See Kölbel (2004) for a similar argument regarding moral judgements, and Schaffer (2011) for discussion pertaining to epistemic modals such as 'might'.
} 
instance, we might say that 'Spinach is tasty' carries a hidden indexical parameter which is supplied by the speaker's standard of taste at the context of use. A consequence of this view is that, in case (1), Bob expresses the proposition Spinach is tasty for Bob (that is, according to Bob's standard of taste), and Carol expresses the proposition Spinach is not tasty for Carol. However, this consequence seems at odds with our judgement that (1) is a disagreement. If the contents of Bob and Carol's utterances are as stated above, there is no conflict between their beliefs: both might be speaking truly, and each could rationally accept the content of the other's belief. The concern, therefore, is that contextualism cannot underwrite the sense in which cases like (1) appear to embody a state of disagreement. The unavailability of an adequate contextualist response to this challenge may give us reason to endorse an alternative treatment of taste-predicates, such as relativism. ${ }^{3}$ (Relativist views will be a central part of our focus later in this paper, and will be outlined in further detail in section three.)

One key fact about the argument from disagreement against contextualism — which is not always acknowledged in the literature - is that it depends crucially on a certain assumption about the nature of disagreement. On a natural interpretation, the argument assumes that there can be no disagreement between subjects where there is no semantic conflict between those subjects' beliefs - i.e. that the beliefs must 'rule each other out' in a strong semantic sense. Because this thought has struck many as irresistible, the argument from disagreement has been taken to be an important objection to contextualism. However, this assumption about disagreement has also been questioned by some who would defend contextualism. Sundell (2011)—whose approach has affinities with my arguments below - argues that once we carefully construe the assumption such as to make it neither mistaken nor question-begging, it is consistent with contextualism. Huvenes (2012), on the other hand, argues that we can understand our intuitions of taste-disagreement in terms of conflict between non-doxastic attitudes rather than in terms of semantic conflict between beliefs, and thus that contextualism avoids the challenge altogether. ${ }^{4}$

\footnotetext{
${ }^{3}$ See (e.g.) Lasersohn (2005) and MacFarlane (2007) for discussion of this move.

${ }^{4}$ Other responses to the argument are also available. One might deny that cases like (1) are instances of disagreement at all (see Cappelen \& Hawthorne (2009: ch.4)). Alternatively, one might, with López de Sa (2008), argue that presuppositions of commonality can generate the relevant kind of conflict even in the absence of semantic conflict. See Baker (2012) for discussion of this and other responses to the argument against contextualism.
} 
My goal in this paper is not to resolve the dialectic surrounding contextualism and disagreement, but rather to explore the role that philosophical assumptions about the nature of disagreement play in arguments from disagreement. My interest in the argument against contextualism is as an exemplar of one kind of argument from disagreement in semantic theory. ${ }^{5}$ Even if the argument does not succeed in undermining contextualism, it highlights an important challenge which contextualists must meet in order for their view to be considered a defensible option for the semantics of taste-predicates. In what follows, then, I assume that the challenge posed by the argument is a legitimate one, and use it as a benchmark for investigating where seemingly illegitimate arguments from disagreement go wrong.

\section{Illicit Assumptions and the Minimal Theory of Disagreement}

Given that arguments from disagreement depend on certain assumptions regarding the nature of disagreement, a key question arises: what kinds of assumptions can legitimately serve as the bases for arguments from disagreement? In order to address this question I will outline and discuss a fictional argument from disagreement which seems uncontroversially illicit. In diagnosing the argument's failure, and distinguishing it from the argument against contextualism, I will explain what we should minimally expect of legitimate arguments from disagreement. The illicit argument in question targets sententialism, the view that sentences, rather than propositions, are the objects of belief. ${ }^{6}$ Here is the argument:

Disagreement between beliefs requires (at least) conflict between propositions believed. As such, sententialism — which holds that beliefs do not express propositions - has the consequence that paradigm cases of disagreement are not, in fact, disagreements. Therefore, sententialism should be rejected.

It is clear that this is an ineffective argument, and that sententialism is not undermined by it. The unimpressive status of this argument can be tracked to the assumption regarding disagreement on

\footnotetext{
${ }^{5}$ There are, of course, other interesting kinds of argument from disagreement. For instance, arguments from disagreement in ethics often charge theories to account for the prevalence of disagreement and inter-cultural divergence rather than to demonstrate consistency with assumptions about disagreement's nature.

${ }^{6}$ See e.g. Higginbotham (2006). For our purposes we should understand sententialism as entailing that propositions play no part in the objects of attitudes.
} 
which it is based - and specifically to the relationship between this assumption and sententialism, the view targeted by the argument. This assumption is as follows:

(D1). Disagreement between beliefs requires conflict between propositions believed.

Importantly, the trouble with D1 it is not that we can easily reject it as false. The orthodox view about semantic content may well have the consequence that something like D1 is true: if propositions are the objects of belief, as is commonly assumed, then it is natural to think that disagreement between beliefs will involve propositional conflict. But nevertheless, this consequence should not concern sententialists. Insofar as sententialists can give an adequate account of disagreement in terms of sentences rather than propositions, they are in a position to deny D1 and instead endorse their alternative account. If sententialists can thereby account for the 'paradigm cases of disagreement' to which the argument refers, they will have discharged their dialectical responsibilities with respect to disagreement. However, propositionalists - the opponents of sententialism who hold that propositions are the objects of belief - are nevertheless well within their rights to endorse D1, a principle which may follow from their theory. Propositionalists are not, however, at liberty to deploy D1 as a premise in an argument against sententialism. We may think it obvious why this is so: that relying on D1 simply begs the question against sententialism unless there is independent motivation for the principle. ${ }^{7}$ However, pinning down the exact nature of this argument's failure will help us to evaluate other arguments whose status as question-begging is disputable. What's more, this diagnosis will allow us to see a useful distinction between two different types of principle regarding disagreement's nature.

To help us to understand the illicit nature of the argument against sententialism, we may consider how it differs from the legitimate challenge to contextualism discussed in section one. The principal difference is as follows: the argument against contextualism does not merely attempt to demonstrate the incompatibility of contextualism with some natural principle regarding disagreement. Rather, in its strongest form, it attempts to show that contextualists have no suitable apparatus with which to fill the theoretical role of disagreement. But nevertheless, as noted previously, the argument still makes a certain assumption regarding disagreement: namely, that

\footnotetext{
${ }^{7}$ If propositionalists can provide some independent argument for D1 which does not rest on propositionalism, this will count against sententialist attempts to provide their own account of disagreement. A parallel version of this point will be relevant in section 4 .
} 
disagreement requires some kind of conflict between the contents of the relevant tastejudgements. Two key questions arise: first, what is the role of this assumption in the argument against contextualism? Second, how does this differ from the role of D1 in the above argument?

I contend that we should take the assumption on which the anti-contextualist argument is based as an attempt to specify a minimal property of doxastic disagreements - that is, as an aspect of the theoretical role of disagreement which all theories must account for. ${ }^{8}$ Theories which cannot account for this feature thereby fail to have adequate explanations of doxastic disagreement. The principle aims to reflect an aspect of the 'minimal account' of disagreement: an account of the phenomenon which is independent of the assumptions and apparatus of any particular semantic theory. ${ }^{9}$ On the other hand, D1 does not appeal to an aspect of the minimal account of disagreement, and this fact explains the difference between the anti-contextualist argument and the anti-sententialist argument.

Although defending a particular minimal theory of disagreement is not part of my project in this paper, the arguments presented below require us to at least have a candidate view on the table. Here I will outline (but not defend) one natural view. As we have seen, the minimal theory cannot require that disagreement must be understood in terms of some specific concept like propositions or sentences, since it seems that either of these options could make for an adequate understanding how disagreement arises. Rather, the minimal theory should highlight the common ground between adequate theory-specific explanations of disagreement. What could play such a role? If we continue to restrict our attention to disagreements involving beliefs, a prime candidate for a minimal theory of disagreement can be given in terms of incompatibility. I rely here on a broad and intuitive notion of incompatibility as a state which precludes joint correctness on some level or other - that the beliefs rule each other out in some way. We are accustomed to understanding incompatibility in terms of preclusion of joint truth - i.e. cases where the beliefs in question

\footnotetext{
${ }^{8}$ As such, the argument against contextualism should be understood as assuming that disagreements like (1) are doxastic disagreements - that they exhibit conflict arising from the subjects' beliefs. Huvenes' (2012) strategy should, therefore, be thought of as denying this assumption and instead claiming that such cases are instances of a different kind of disagreement: namely, disagreement in attitude. Understood this way, his response is consistent with endorsing the minimal theory of doxastic disagreement outlined here.

${ }^{9}$ This idea has affinities with the work of Greenough (2003: 235), whose minimal theory of vagueness aims to 'give a rigorous characterization of vagueness from a perspective which is as neutral as possible on matters logical and philosophical'.
} 
cannot all be true - and it is clear that this standard notion is often assumed in philosophical discussions about disagreement. In order to be of use to us here, however, the notion of incompatibility at work must be broader than this — specifically, it must leave room for all theories which have access to some positive explanation of how doxastic disagreement arises. As such, we cannot insist that the notion be understood in terms of preclusion of joint truth, since there are other kinds of genuine conflict arising from subjects' beliefs which are worthy to be described as incompatibilities. To give one example, this minimal theory is consistent with the way in which shared presuppositions can give rise to a conflict between beliefs whose contents would otherwise be consistent (see López de Sa (2008)) — cases in which incompatibility between beliefs arises only in the light of aspects of context. ${ }^{10}$

There is much to be said about how, precisely, this minimal theory of disagreement should be understood. However, extensive discussion here would take us too far off course from the dialectic of this paper, and as such my discussion will be based on the loose form of the view described above. Furthermore, one might doubt that an incompatibility account of disagreement can play the minimal role required here. However, since I am using the incompatibility view to illustrate my arguments, rather than defending the view, discussion of these concerns will be postponed for another time.

Let us return to the dialectic between propositionalists and sententialists. Crucially, the minimal theory of disagreement as incompatibility admits of obvious concrete interpretations in terms of either view — and, moreover, this would presumably also be true of any other plausible candidate for a minimal theory of disagreement. We can think of D1, under certain assumptions, as a consequence of conjoining the incompatibility view with the propositionalist doctrine that propositions are the objects of belief. Let us call principles like D1- those which result from a conjunction of the minimal account with the assumptions and/or apparatus of some particular theory - 'loaded principles', since that they are loaded with the apparatus and/or assumptions of a particular theory. Every semantic theory may endorse loaded principles regarding disagreement in this sense. However, where two competing theories like propositionalism and sententialism

\footnotetext{
${ }^{10}$ Furthermore, this minimal view of doxastic disagreement must also be broad enough to avoid falling foul of Sundell's (2011: $280 \mathrm{fn}$. 20) concerns about MacFarlane's (2007) 'preclusion of joint accuracy' account of disagreement. To take just one of Sundell's examples, conflicts arising from metalinguistic expression rather than literal expression will be sufficient to count as incompatibilities in this sense.
} 
endorse inconsistent loaded principles, we need not necessarily think that they disagree about the minimal account of disagreement. In a debate about what constitutes disagreement, propositionalists and sententialists are perfectly at liberty to agree that disagreement arises as a result of incompatibility between beliefs. As such, their divergence over principles like D1 does not, in one sense, really concern disagreement at all. Rather, it is a consequence of the fact that their respective theories disagree about what the bearers of incompatibility are - and as a result they describe the same minimal view of disagreement in different 'loaded' terms.

In the light of the distinction between loaded principles and the minimal theory of disagreement, we can develop a dilemma for the argument against sententialism. Insofar as the sententialist can give an alternative to D1- that is, some explanation of how incompatibility, and therefore disagreement, arises in terms of sentences rather than propositions - the argument simply fails to highlight an explanatory deficiency in sententialism. But if the sententialist cannot give such an explanation, the theory's conflict with D1 is merely a symptom of sententialism's broader problems with disagreement; namely, that the view has no resources with which to account for how disagreement arises in accordance with the minimal account. So the dilemma is as follows: either the argument's key charge is mistaken, if sententialists can account for disagreement; or it is misplaced, since a stronger charge against sententialism could be made. The argument against contextualism, on the other hand, does not face this dilemma: if contextualists cannot give an adequate account of disagreement in cases like (1) then the theory's conflict with the argument's premises - i.e. contextualism's clash with the minimal theory of disagreement - is a full representation of the explanatory deficiency of the theory.

The contrast between these two arguments allows us to derive key methodological conclusions about how arguments from disagreement should be formulated. First, a negative principle: arguments from disagreement should not take 'loaded' principles, such as D1, as their premises. Arguments based on such principles will be dialectically hopeless, since a conflict with a loaded account of disagreement is not in itself a reason to reject a theory. Such arguments are therefore liable to face dilemmas like the one outlined above. As such, we should not think of loaded philosophical theories and assumptions regarding disagreement as part of the data which must be accommodated by semantic theories. 
On the other hand, conflict with the minimal theory of disagreement — characterised by an inability to account for the basic theoretical role of doxastic disagreement - is a compelling reason to reject a theory. Thus, arguments from disagreement will be legitimate if they form their main premises by appeal to some aspect of the minimal theory of disagreement. Such arguments represent more than a challenge to endorse some parochial description of disagreement in terms of a particular theory's assumptions; rather, they represent a basic challenge to provide any apparatus with which to account for our judgements of disagreement. The argument against contextualism is best understood in these terms: as an attempt to demonstrate the inability of contextualism to account for a key aspect of disagreement which all theories are required to underwrite. ${ }^{11}$ Similarly, a legitimate argument from disagreement against sententialism, if one were available, would have to take this form. ${ }^{12}$ The minimal theory of disagreement should, therefore, be considered part of the raw data to which semantic theories are answerable.

The methodological principles described here purport to show that certain arguments from disagreement are dialectically ineffective. But one might think, with Williamson (2007), that dialectically inefficacy is not a fatal flaw in an argument, since evidence neutrality fails. That is: since legitimate philosophical evidence need not always be recognised as such by one's opponents, an argument should not be dismissed merely because it cannot convince one's opponents. ${ }^{13}$ However, those who reject evidence neutrality should arguably still prefer to employ arguments which are dialectically effective over those which are not, where the choice is available, even if dialectically effective arguments are not strictly required to bolster their positions. As such, insofar as this paper succeeds in showing that some arguments from disagreement are dialectically effective, this at least shows that these should be preferred over arguments from disagreement

\footnotetext{
${ }^{11}$ To reiterate, we should also understand these arguments as appealing to the premise that disagreements in relevant domain are doxastic disagreements — an assumption which will, in some domains, be up for debate.

${ }^{12}$ Appealing to an aspect of the minimal theory will not, strictly, be a necessary condition on legitimacy for such arguments. Assuming a principle which is broad enough to be binding upon a certain theory - even if that principle is not also general enough to be part of the minimal account of disagreement — will be sufficient for a legitimate challenge. If a theory is bound by a certain principle then adherence to that principle is a necessary condition for the theory to give any adequate positive account of disagreement. In this sense, a theory's being bound by a principle does not entail that the principle will be part of the minimal theory of disagreement. However, since it may not always be easy to determine which principles a theory is bound by, the 'safest' arguments from disagreement will be those which appeal only to aspects of the minimal theory.

${ }^{13}$ Cappelen \& Hawthorne (2011b) advert to this view in a related discussion.
} 
which fail to be dialectically effective - even if it does not show that the dialectically ineffective arguments should be rejected altogether.

\section{Cappelen \& Hawthorne's 'Agree-Based Diagnostic'}

This section introduces another argument from disagreement - this time, an argument against relativism. Herman Cappelen \& John Hawthorne (2009) argue that we can appeal to reports of agreement and disagreement to detect the presence and absence of context-sensitivity - and, consequently, that we can assess the adequacy of certain theories in terms of such reports. In this vein, Cappelen \& Hawthorne (hereafter ' $\mathrm{C} \& \mathrm{H}$ ') present three 'Agree-Based Diagnostics' for context-sensitivity. One of these diagnostics concerns disagreement-reports rather than agreementreports - and this disagreement-based diagnostic will be our focus here. The diagnostic is as follows:

Let $\mathrm{u}$ be a sincere utterance of (a sentence) $\mathrm{S}$ by (a subject) A in (a context) $\mathrm{C}$ and $\mathrm{u}$ ' a sincere utterance of not-S by (a second subject) B in (a second context) C'. If from

a third context C' they cannot be correctly reported by 'A and B disagree whether $\mathrm{S}^{\prime}$, then S is semantically context sensitive. (C\&H 2009: p.54)

$\mathrm{C} \& \mathrm{H}$ argue that this diagnostic conflicts with relativist semantic theories, since relativists are committed to the absence of context-sensitivity in cases which meet the antecedent conditions of the diagnostic. This section outlines outline C\&H's argument from disagreement based on the above diagnostic and show that it rests on a certain principle regarding the nature of disagreement. Section four uses the methodological principles discussed in section two to show that their argument cannot be dialectically effective. Specifically, I will show that that C\&H's argument fits the pattern of the illicit argument against sententialism discussed above, since the assumption regarding disagreement on which their argument rests makes it similarly dialectically ineffective.

To illustrate C\&H's argument, consider relativism about weather-reports. ${ }^{14}$ Proponents of this view hold that expressions like 'it is raining' vary in truth conditions between contexts, but that this

\footnotetext{
${ }^{14}$ Note that this view is not sufficient for relativism in MacFarlane's sense of assessment-sensitivity. Rather, for MacFarlane, weather-report relativism is naturally understood as a species of 'non-indexical contextualism'. See MacFarlane (2009).
} 
variation cannot - contrary to the contextualist view about such matters - be traced back to divergence in semantic content between contexts. Relativists hold that 'it is raining' is invariant between locations, in that it expresses the same proposition no matter where it is used-and, as such, that weather-reports are not context-sensitive with respect to location. But since the weather clearly does vary between locations, the intuitive variation in truth conditions for 'it is raining' must be accounted for in another way. Accordingly, relativists hold that the locationneutral proposition it is raining can only be evaluated for truth with respect to some location or other. Relativists, then, propose adding a location-parameter to the circumstance of evaluation against which propositions are assessed for truth—so a single proposition might be true at some locations and yet false at others. With this view in mind, consider case (2):

$$
\begin{array}{ll}
\text { (2) Bob: 'It's raining.' } & \text { (Location: London) } \\
\text { Carol: 'It's not raining.' } & \text { (Location: Manchester) }
\end{array}
$$

Relativists hold that the proposition expressed by Carol is the negation of the proposition expressed by Bob, since weather-reports are invariant with respect to location. But although the propositions they express are location-neutral, Bob and Carol's respective contexts nevertheless determine privileged location-parameters against which their individual utterances should be evaluated for truth — and in paradigm cases, the privileged location determined by context will be the location of the speaker. Call this - the privileged location determined by context - the location which the utterance concerns. In this sense, Bob's utterance concerns London, and one should evaluate his utterance for truth only according to the weather in London. ${ }^{15}$ Similarly, Carol's utterance concerns (and thus inherits its truth conditions from) Manchester. Now suppose that it is indeed raining in London and dry in Manchester. Under this supposition, the proposition Bob expresses in (2) is true in London yet false in Manchester, and vice versa for Carol—and so their respective utterances are each true according to the privileged locations they concern.

However, we should not think that Bob and Carol disagree in (2). There is no 'semantic clash' between their utterances: it is perfectly consistent that it be raining in London while it is not raining in Manchester, and despite the superficial contradiction in (2) there seems to be no sense in which their utterances rule each other out. But this judgement puts relativism at odds with

\footnotetext{
${ }^{15}$ This discussion abstracts away from the further question of temporal relativity in weather-reports.
} 
$\mathrm{C} \& \mathrm{H}$ 's agree-based diagnostic. Recall C\&H's claim: if, in a case where one subject utters $\mathrm{S}$ and a second subject utters not-S, the two cannot be reported as disagreeing, then $\mathrm{S}$ is contextsensitive. Since Case (2) meets the antecedent conditions of this diagnostic, C\&H (2009: 97) would have us infer that 'it is raining' is semantically context-sensitive. This contradicts the key tenet of weather-report relativism - namely, that weather-reports are invariant - and so the agree-based diagnostic, in conjunction with our judgement about case (2), entails that weatherreport relativism is false.

Like the arguments from disagreement discussed in sections one and two, the agree-based diagnostic rests on a certain assumption about what disagreement consists in. The assumption in question, I contend, is D2:

(D2). A and B disagree about a proposition $p$ if one believes it and one believes the negation. ${ }^{16}$

This principle is, given natural assumptions, equivalent to the contrapositive of the relevant clause in the agree-based diagnostic - so one cannot accept the agree-based diagnostic whilst rejecting D2. To help illustrate this, consider first the relevant clause in the diagnostic, ABD (for 'AgreeBased Diagnostic'):

(ABD) If from a third context C' (A and B) cannot be correctly reported by 'A and B disagree over $\mathrm{S}^{\prime}$, then $\mathrm{S}$ is semantically context-sensitive. ${ }^{17}$

Assume the plausible principle that if A and B do not disagree, then there is no context in which they can be correctly reported as disagreeing. By transitivity, this allows us to infer the following:

(ABD2) If $\mathrm{A}$ and $\mathrm{B}$ do not disagree over $\mathrm{S}$, then $\mathrm{S}$ is semantically context-sensitive.

The contrapositive of this, allowing a double-negation elimination, is as follows:

\footnotetext{
${ }^{16} \mathrm{C} \& \mathrm{H}$ themselves endorse this principle in a later discussion: C\&H (2011a: p.452).

${ }^{17}$ Note that (ABD) is itself the consequent of another conditional whose antecedent is 'A utters $\mathrm{S}$ in one context and $\mathrm{B}$ utters not-S in another context'. This will be important below.
} 
(CP-ABD2) If $\mathrm{S}$ is not semantically context-sensitive then $\mathrm{A}$ and $\mathrm{B}$ disagree over $\mathrm{S}$.

Given C\&H's description of the case, the transition from CP-ABD2 to a version of D2 is smooth. If $\mathrm{A}$ utters $\mathrm{S}$ and $\mathrm{B}$ utters not-S, and $\mathrm{S}$ is not context-sensitive, it is natural to infer that $\mathrm{A}$ expresses a proposition and B expresses its negation. If we name this proposition ' $p$ ', and assume that $\mathrm{A}$ and $\mathrm{B}$ are sincere, the case is equivalent to one in which $\mathrm{A}$ believes $p$ and $\mathrm{B}$ believes not- $p$. This allows us to derive D2*:

$(\mathrm{D} 2 *)$ If $\mathrm{A}$ believes a proposition $\mathrm{p}$ and $\mathrm{B}$ believes its negation, then $\mathrm{A}$ and $\mathrm{B}$ disagree about $p$.

D2* is merely an alternately-phrased version of D2. The upshot is that the agree-based diagnostic - and the argument against relativism — cannot be effective if the view of disagreement described in $\mathrm{D} 2$ is problematic.

\section{The Diagnostic's Failure}

This section uses the methodological principles discussed in section two to argue that D2 is an unsuitable principle on which to base an argument against relativism - and, since the central principle of the diagnostic entails D2, that C\&H's use of the agree-based diagnostic against relativism is illicit. ${ }^{18}$

First, consider how a relativist should respond to the argument based on ABD. One way to rebut the argument is to develop an alternative principle regarding disagreement which avoids the problematic entailment that (2) is a disagreement. That is, a relativist should explain how their theory is consistent with our judgement that (2) is not a disagreement. If such an explanation is available, then the relativist may be in a position to reject D2 in favour of an alternative principle. If this is so, then conflict with D2 - and thus with the agree-based diagnostic which entails D2 does not represent a troubling objection to relativism. Of course, this is not a trivial move: there

\footnotetext{
${ }^{18}$ MacFarlane (2011: 448 fn. 8) briefly makes a similar point against C\&H. Here I aim to give a fuller explication of the argument's failure. Caponigro \& Cohen (2011) offer a different critique of C\&H's argument.
} 
is a serious question over whether any adequate relativist replacement for D2 can be provided. To help us illustrate how the dialectic should proceed, however, let us consider a candidate weatherreport relativist alternative to $\mathrm{D} 2$ :

(D3) If A believes $p, \mathrm{~B}$ believes not-p, and A's belief and B's belief both concern the same location, then $\mathrm{A}$ and $\mathrm{B}$ disagree about $p$.

In a reply to Richard (2011), who proposes a variant of D3 on behalf of relativists, $\mathrm{C} \& \mathrm{H}$ argue that any move to deny D2 in favour of an alternative principle like D3 faces unacceptable costs. Their argument will be addressed later in this section, after we have explored how the availability of a principle like D3 would undermine C\&H's argument.

D3 is consistent with the absence of disagreement in (2), since in that case the relevant beliefs do not concern the same location: Bob's belief concerns London while Carol's concerns Manchester. Insofar as D3 or some other relativist principle is an adequate explanation of disagreement involving relativistic contents more generally, then, we should not take the clash between relativism and D2 (and the diagnostics which entail D2) to be a dialectical problem for relativists, since both D2 and D3 represent acceptable ways of understanding the notion of disagreement. In sum: assuming that relativists have access to some account of disagreement shows the agree-based diagnostic to be entirely ineffectual. ${ }^{19}$

The dialectical situation here matches the state of play between propositionalists and sententialists discussed previously. Both D2 and the relativist alternative, D3, appear to be consistent with a common minimal understanding of disagreement in terms of incompatibility - that is, both views can be understood as interpretations of that minimal view in terms of the apparatus of the respective theories. Given Cappelen \& Hawthorne's orthodox assumptions regarding monadic truth and propositions, it seems to follow that clashing propositions must always give rise to incompatibility in the traditional sense-and thus, as encapsulated in D2, that a subject who believes $p$ must be in disagreement with a subject who believes not- $p$. However, given the relativist view of propositions and attitudes, superficially contradictory propositions need not

\footnotetext{
${ }^{19}$ Below I will consider what follows if we reject the assumption that D3 (or an alternative principle) can successfully explain the relevant cases in relativist terms.
} 
always give rise to incompatibility - rather, the presence of this state will depend on the parameters which particular occurrences of the propositions concern. As such, a principle which further details under what conditions clashing propositions give rise to incompatibility, like D3, is required. But note that, for any arbitrary case, $\mathrm{C} \& \mathrm{H}$ and the relativists agree about both whether that case exhibits incompatibility and whether that case exhibits disagreement. What they disagree about in each case is the specific way in which objects of belief in context contribute to incompatibility and disagreement. So, in the sense outlined above, both D2 and D3 are 'loaded' principles regarding disagreement — both principles build in assumptions from specific theories. But since these principles are consistent with a common understanding of what disagreement is namely, that doxastic disagreement arises from incompatibility — we need not think that $\mathrm{C} \& \mathrm{H}$ and the relativists disagree about the nature of disagreement. Their differences over principles like D2 and D3 can instead be traced back to the more basic conflicts between their respective commitments in semantic theory.

If relativists have access to some alternative principle like D3 with which to account for the disagreement-data, then the agree-based diagnostic fails since relativists can meet the challenge it poses. But as noted above, it is far from clear that this will be the case: determining whether relativists can provide an explanation of disagreement which is consistent with the minimal theory is a significant project in itself. So we must also consider what follows for the agree-based diagnostic if relativists cannot supply an adequate principle in the mould of D3 - if no such alternative is available. Even under this assumption, however, we should not think that C\&H's argument is effective. If relativists cannot account for the presence and absence of disagreement, then the argument based on D2 will be misplaced: the relevant charge against relativism will not merely be that it is inconsistent with $\mathrm{D} 2$, but rather that it has no resources with which to account for the theoretical role of disagreement in semantic theory. That is, the objection will not be that relativists cannot accept some particular loaded principle like D2, but rather that they cannot explain how incompatibility arises at all in central cases of disagreement and so cannot endorse even the minimal theory of disagreement. The thought here is as follows: if relativists face a fundamental explanatory deficiency regarding disagreement, then merely adverting to the inconsistency with some particular loaded principle regarding disagreement is not a useful way to express the problem. 
Like the argument against sententialism, then, the use of the agree-based diagnostic against relativism faces a dilemma. On the first horn, relativists can explain how their view is consistent with the minimal theory of disagreement, whether through D3 or some other principle; if this is so then C\&H's argument fails to highlight an explanatory deficiency of relativism. On the second horn, relativists cannot give an explanation of disagreement; if this is so then the argument fails to identify the real explanatory problem facing relativists - namely, that they cannot account even for the minimal theory of disagreement. If relativists cannot give any account of disagreement consistent with the minimal theory, then highlighting a conflict with some particular parochial account of disagreement such as D2 is an objectionably weak and potentially misleading way to express this deficiency; but insofar as one lacks reason to think that relativists cannot give an account of disagreement, the charge based on the agree-based diagnostic has no force. Given this dilemma, Cappelen \& Hawthorne's argument cannot be dialectically efficacious against relativism. ${ }^{20}$ This is an instance of the general principle that arguments based on loaded principles will be dialectically hopeless.

Earlier I noted that C\&H anticipate one key move in my argument - specifically, that they object to the idea that relativists can deny D2 and endorse an alternative loaded principle regarding disagreement. The thought is this: if we have significant independent reason to think that D2 is irresistible - that C\&H's loaded principle must be accepted - then we may, after all, be in a position to wield the agree-based diagnostic against relativism, since evidence for D2 is also evidence against any alternative relativist principle which, like D3, is inconsistent with D2. C\&H appear to believe that independent reason to accept their loaded principle does indeed existand, specifically, that any attempt to deny D2 incurs significant dialectical costs. Their objection is as follows:

Suppose one is a relativist who (...) thinks that some additional criteria need to be met for disagreement-judgments to be correct beyond the relevant pattern of belief and denial (i.e. D2). Then it is a short step to endorsing speeches of the following sort: 'She believes that what I believe is false, but she doesn't disagree with me'. But that speech sounds terrible. (C\&H 2011a: 452-343)

\footnotetext{
${ }^{20}$ Theorists who think that dialectically ineffective arguments are not fatally flawed should, as argued above, nevertheless prefer to endorse the alternative (dialectically legitimate) challenge to relativism arising from this dilemma - a challenge which is described below.
} 
While there may be an objection to relativism here, I contend that it is not a challenge to the cogency of D2-denying strategies since it does not specifically concern disagreement. To illustrate, recall the weather-report exchange in case (2). Bob, in London, believes that it is raining - whilst Carol, in Manchester, believes that it is not. In the light of this case, consider a speech similar to that discussed by $\mathrm{C} \& \mathrm{H}$ above:

(BF1) Bob: 'She believes that what I believe is false.'

Now either BF1 is acceptable, or it is not. Either way, it seems, C\&H's argument fails to show that D2 is irresistible. First, suppose that BF1 is unacceptable; that, in C\&H's words, it too 'sounds terrible'. If this is so, then the relativist's commitment to the truth of BF1 represents the basis for a legitimate objection to the theory. But if this is so, then C\&H's point has been made using an example which does not concern disagreement at all. This suggests that the trouble here does not stem from the relativist's denial of $\mathrm{D} 2$, but is rather a more general problem for relativists concerning correct belief-reports and intuitions about the contents of beliefs. ${ }^{21}$ If BF1 is unacceptable, it is because the statement contravenes an intuition about the dissimilarity of the content of Bob and Carol's beliefs. This is a serious objection to relativism, to be sure, but it does not appear to rule out the relativist's strategy of denying D2 and providing an alternative account of disagreement.

On the other hand, suppose that BF1 is acceptable. Given that the clause of C\&H's example which is omitted from BF1 is true - namely, that the subjects do not disagree in the case in questionthis presumably means that Bob should also be able to describe the case in terms of BF2, C\&H's original 'terrible speech':

(BF2) Bob: 'She believes that what I believe is false, but we don't disagree.'

If both $\mathrm{BF} 1$ and $\mathrm{BF} 2$ are acceptable, then $\mathrm{C} \& \mathrm{H}$ have no case against the relativist here. But if BF1 is acceptable whilst BF2 is not, then the unacceptability of BF2 must be related to some pragmatic

\footnotetext{
${ }^{21}$ There is a parallel here to problematic examples for temporalism discussed by Richard (1981). Richard's examples appeal to intuitions about the contents of beliefs across times, and wields them against views which postulate temporally neutral contents — while the putative objection to BF1 here appeals to intuitions about the contents of beliefs across locations and wields them against views which postulate locationally-neutral contents.
} 
infelicity rather than to its truth value. But since the objection here was supposed to be that relativists are committed to the truth of this statement, the admission of a pragmatic infelicity in $\mathrm{BF} 2$ provides a clear way for relativists to address the objection. Specifically, they can attempt to give a fuller pragmatic explanation of why the conjunction of the (acceptable) BF1 with the (true) extra clause yields an unacceptable statement, BF2. ${ }^{22}$ As such, it cannot be a fatal cost for relativism that it is committed to BF2's truth - and so C\&H's objection fails. Once we consider a concrete case of $\mathrm{C} \& \mathrm{H}$ 's objection, there is no interpretation on which it represents a challenge specifically to the relativist account of disagreement. C\&H fail, therefore to show that a strategy which denies D2 is unavailable to the relativist.

One might object here by claiming that the trouble with BF1 cannot be so cleanly separated from disagreement - that is, by questioning my claim that the objection to BF1 can be stated without reference to disagreement. More specifically, one may think that our intuitions about Bob and Carol's failure to share content in BF1 arise because we judge that the two subjects do not disagree, and thus that BF2 is simply a more precise formulation of what was wrong with BF1 all along. If this is correct, then $\mathrm{C} \& \mathrm{H}$ 's defence of the idea that $\mathrm{D} 2$ is irresistible may be rescued, since BF1 does after all highlight a cost associated with denying D2.

However, this objection appears to take for granted the central contested move at the heart of the agree-based diagnostic: namely, that where we judge an absence of disagreement in a case where one subject utters $\mathrm{S}$ and a second subject utters not-S, we have sufficient grounds to conclude that the two subjects do not share content. As such, the objection does not provide reason to think that D2 is irresistible beyond what is already contained in the agree-based diagnostic - since it is, after all, already common ground that the agree-based diagnostic entails D2. Furthermore, since my arguments aim to show that $\mathrm{C} \& \mathrm{H}$ cannot legitimately appeal to the agree-based diagnostic in arguing against relativism, it follows that a defender of $\mathrm{C} \& \mathrm{H}$ cannot establish the irresistibility of $\mathrm{C} \& \mathrm{H}^{\prime}$ 's underlying assumptions regarding disagreement by appeal to a principle on which the diagnostic relies. In order for C\&H's goal of showing that relativists should not reject D2 to be achieved in a dialectically potent manner, we require some reason to accept D2 which is not

\footnotetext{
${ }^{22}$ Furthermore, even if C\&H can show that denying D2 and accepting BF2 represents a dialectical cost, it does not follow that such strategies can be ruled out altogether. Dialectical costs can be traded against dialectical benefits elsewhere, and the existence of a particular dialectical cost does not entail that all strategies which deny D2 are unacceptable ways of detailing the minimal theory of disagreement in 'loaded' relativist terms.
} 
already bound up with the principles that constitute the agree-based diagnostic - and the response here does not provide such independent reason.

It should be reiterated that my arguments leave open an alternative argument from disagreement against relativism which adheres to the methodological principles discussed above. ${ }^{23}$ This remnant objection should be thought of as parallel to the challenge to contextualism described earlier. This corresponds to the doubt, already mentioned above, over whether D3 - or any other candidate relativist principle regarding disagreement - can adequately fulfil the required role and underwrite the correct verdicts in paradigm cases. Relativists, then, must explain how their theory has the resources to explain the basic role of disagreement in semantic theory. Relativists about matters of taste, for instance, must - insofar as they hold that disagreement about taste is a matter of doxastic disagreement - explain how we are to understand incompatibility in a relativist framework in order to deliver the right predictions about basic taste-disagreements like case (1). ${ }^{24}$ Such an argument, which is based on the minimal theory of disagreement, rather than a loaded principle like D1, represents a serious challenge - and one which it is far from clear that all relativist theories can respond to. The failure of the agree-based diagnostic, then, does not entail that relativism is out of the woods with respect to disagreement.

\section{Philosophical Assumptions in Semantic Theory: Taking Stock}

I have attempted to show that Cappelen \& Hawthorne's argument against relativism, like the fictional argument against sententialism, faces a dilemma: either it falls flat (if relativists can give an adequate alternative account of disagreement), or it fails to highlight what is really wrong with relativism (if relativists cannot give such an account). More generally, however, my arguments bear on questions about the role of philosophical assumptions about disagreement in semantic theory - that is, about what kinds of assumptions should be considered part of the data for which semantic theories can be held to account - and questions concerning the broader relationship between semantic and philosophical theorising about disagreement.

\footnotetext{
${ }^{23}$ See e.g. Stojanovic (2007) for more discussion of relativism's troubles with disagreement.

${ }^{24}$ While the weather-report relativist may accept D3, we should note that a similar principle applied to relativism about taste seems to deliver the same problematic result which supposedly follows from contextualism: namely, that where subjects have differing standards of taste, they do not disagree.
} 
The upshot of my arguments is as follows. First, loaded philosophical principles regarding disagreement - theories and assumptions which go beyond a minimal picture of the concept and, for example, detail precisely which mental relations can constitute disagreement-cannot typically be utilised in dialectically effective arguments against semantic theories. As such, these loaded principles should not be considered part of the data to which semantic theories are answerable, and one should not attempt to scrutinise semantic theories on the basis of such principles. On the other hand, the minimal account of disagreement should be considered part of the basic data for constructing and evaluating semantic theories. Theories must account for intuitive predications regarding the attribution of states of disagreement to subjects in virtue of their beliefs in a way consistent with this minimal theory. A semantic theory which can adequately demonstrate its consistency with the existence of disagreement in this minimal, theory-neutral sense - be it understood in terms of incompatibility, as suggested above, or in other terms - will have discharged its dialectical responsibilities with respect to disagreement. Above I claimed that the influential argument from disagreement against contextualism should be understood as appealing to the minimal theory of disagreement: as a basic challenge to contextualists to explain how their view is, contrary to appearances, consistent with intuitive predictions of disagreement in line with the minimal view. C\&H's argument against relativism, however, has been shown to rest illicitly on loaded philosophical principles regarding disagreement's nature.

Since I contend that arguments from disagreement based on loaded philosophical principles are illicit, one might think that this indicates a fundamental disconnect between semantic theories and philosophical theories of disagreement - and, perhaps, that it is suggestive of a general isolation of semantic theorising about linguistic content from philosophical theorising about mental content. However, it is worth noting that my arguments do not have this consequence. To reiterate, my claim is not that inferences from loaded philosophical assumptions regarding disagreement to linguistic conclusions in semantic theory are necessarily unsound. ${ }^{25}$ Nothing proposed here rules out the possibility of sound arguments from substantive philosophical premises about disagreement to semantic conclusions in linguistic theory - and as such the two domains are not isolated in principle. In practice, however, arguments based on loaded principles regarding

\footnotetext{
${ }^{25}$ Recall, for instance, the argument against sententialism. A key point in the evaluation of this argument is that we may not be in a position to rule out the key premise, D1, as false - and so we may not be in a position to prove that the argument is unsound - but we can nevertheless show that the argument is illicit since it rests on a loaded principle regarding disagreement. The same is true of D2's role in C\&H's argument against relativism.
} 
disagreement should be rejected, since — for reasons described previously — they will fail to be dialectically effective against semantic theories.

As we have seen, there are also additional ways in which semantic approaches to disagreementthat is, the attempts of theorists to demonstrate consistency with the minimal theory-are open to philosophical scrutiny. Recall that $\mathrm{C} \& \mathrm{H}$ attempt to screen off the possibility of a relativist response to their argument by arguing that alternative principles regarding disagreement like D3 are inherently untenable. Their argument fails, but — unlike their initial argument - its purpose is not illegitimate. If such an argument were to succeed, relativists would be required to return to the drawing board in their attempts to account for disagreement. Similarly, certain attempts to undermine certain contextualist responses to the argument from disagreement (e.g. MacFarlane (2007); Baker (2012)) will also fall into this category. Such strategies further indicate that semantic and philosophical theorising about phenomena such as disagreement should not be considered fundamentally separate. Crucially, however, such arguments must - like arguments from disagreement themselves - avoid premises based on loaded philosophical assumptions regarding disagreement, lest they also be dialectically ineffective.

This paper has assumed a minimal account of doxastic disagreement understood in terms of incompatibility. While I take this account to be not only plausible, but also implicit in much of the relevant literature, my appeal to the account has been for illustrative purposes and as a placeholder for a wider debate. As such, further research into the precise nature of the minimal theory of disagreement - and what exactly can be taken as data for a semantic theory to explainwill help us to shed further light on the role of disagreement in semantic theory. ${ }^{26}$

\section{References}

Baker, C. 2012. Indexical Contextualism and the Challenges from Disagreement, Philosophical Studies 157/1: 107-123.

Caponigro, I. \& Cohen, J. 2011. On collection and covert variables, Analysis 71/3: 478-488.

Cappelen, H. \& Hawthorne, J. 2009. Relativism and Monadic Truth. Oxford: Oxford University Press.

Cappelen, H. \& Hawthorne, J. 2011a. Reply to Lasersohn, MacFarlane and Richard, Philosophical Studies 156/3: 449-466.

Cappelen, H. \& Hawthorne, J. 2011b. Reply to Glanzberg, Soames and Weatherson, Analysis 71/1: 143156.

\footnotetext{
${ }^{26}$ Earlier versions of this paper were presented at the University of Leeds, the Northern Institute of Philosophy at the University of Aberdeen, and the 3rd Workshop on Semantic Content and Context Dependence at the University of Barcelona. I am grateful for feedback received on these occasions. For helpful comments and suggestions, thanks are also due to Jon Robson, Aaron Meskin, and Matthew Kieran.
} 
Greenough, P. 2003. Vagueness: A Minimal Theory, Mind 112/446: 235-281.

Higginbotham, J. T. 2006. Sententialism: the Thesis That Complement Clauses Refer to Themselves. Philosophical Issues 16/1: 101-119.

Huvenes, T. T. 2012. Varieties of Disagreement and Predicates of Taste, Australasian Journal of Philosophy 90/1: 167-181.

Kölbel, M. 2004. Indexical Relativism versus Genuine Relativism, International Journal of Philosophical Studies 12/3: 297-313.

Lasersohn, P. 2005. Context dependence, disagreement, and predicates of personal taste, Linguistics and Philosophy 28/6: 643-686

López de Sa, D. 2008. Presuppositions of Commonality, In Relative Truth,ed. Max Kölbel and Manuel García-Carpintero. Oxford: Oxford University Press: 297-310.

MacFarlane, J. 2007. Relativism and Disagreement, Philosophical Studies 132/1: 17-31.

MacFarlane, J. 2009. Nonindexical Contextualism, Synthese 166/2: 231-250.

MacFarlane, J. 2011. Simplicity Made Difficult, Philosophical Studies 156/3: 441-448.

Richard, M. 1981. Temporalism and Eternalism, Philosophical Studies 39/1: 1-13.

Richard, M. 2011. Relativistic Content and Disagreement, Philosophical Studies 156/3: 421-431.

Schaffer, J. 2011. Perspective in Taste Claims and Epistemic Modals, in Epistemic Modality, ed. Andy Egan \& Brian Weatherson. Oxford: Oxford University Press: 179-226.

Stojanovic, I. 2007. Talking about taste: disagreement, implicit arguments, and relative truth, Linguistics and Philosophy 30/6: 691-706.

Sundell, T. 2011. Disagreements About Taste, Philosophical Studies 155/2: 267-288.

Williamson, T. 2007. The Philosophy of Philosophy. Oxford: Oxford University Press. 